Research Paper

\title{
DICER 1 regulates endometrial carcinoma invasion via histone acetylation and methylation
}

\author{
Bilan Li, Wen Lu, Junjie Qu, Yongli Zhang, Xiaoping Wan ${ }^{\bowtie}$ \\ Department of Gynecology, Shanghai First Maternity and Infant Hospital, Tongji University, School of Medicine, Shanghai, P.R. China. \\ $\bowtie$ Corresponding author: Xiaoping Wan, E-mail: Xiaopingwan33@163.com. \\ (C) Ivyspring International Publisher. This is an open access article distributed under the terms of the Creative Commons Attribution (CC BY-NC) license \\ (https://creativecommons.org/licenses/by-nc/4.0/). See http://ivyspring.com/terms for full terms and conditions.
}

Received: 2016.09.01; Accepted: 2016.11.10; Published: 2017.03.12

\begin{abstract}
Endometrial carcinoma $(E C)$ is one of the most common gynecologic malignancy, but molecular mechanisms of the development and progression of EC remain unclear. Here we showed that the expression of DICERI was negatively associated with the level of histone methylation, histone acetylation and PRC2 components SUZ12 and EZH2 in EC cells. In addition, knockdown of DICERI significantly downregulated miR-200b and let-7i, which may then regulate their targets SUZ12 and EZH2. Furthermore, knockdown of DICER1 remarkably suppressed the expression of epithelial cell marker E-cadherin, induced the expression of mesenchymal cell marker Vimentin, and promoted the invasion of EC cells. In conclusion, our data suggest that DICERI suppresses SUZ12 and EZH2 via affecting their upstream miRNA synthesis, and inhibits epithelial-mesenchymal transition(EMT) and invasion of EC cells via histone modification.
\end{abstract}

Key words: DICER1; endometrial carcinoma; histone methylation; histoneacetylation; epithelial-mesenchymal transition

\section{Introduction}

Endometrial carcinoma (EC) is the fourth most common cancer in the women worldwide [1,2]. Despite advances in cancer therapies, the incidence of endometrial carcinoma increases. In particular, the prognosis is poor for the patients with recurrent or advanced EC. Recent evidences indicate that epigenetic mechanisms are involved in the development and progression of cancer [3]. DNA methylation and histone modifications are important epigenetic modifications that could control gene expression [4]. There are at least eight patterns of histone modifications, including methylation and acetylation [5]. Histone methylation and acetylation are reversible and thus could modulate chromatin structure to impact gene expression [6,7]. Currently, global patterns of histone modifications are still unknown in EC [8]. SUZ12 and EZH2 are two histone H3K27 methyltransferases that mediate transcriptional repression [9].

MicroRNAs (miRNAs) are short non-coding RNAs that regulate the expression of almost $30 \%$ genes [10-12]. DICER1 regulates the processing of all endogenous small interference RNAs (siRNAs) and miRNAs. Down-regulation of DICER1 in breast cancer was related to miR-200/epithelialmesenchymal transition (EMT) and cancer cell metastasis [13].

In this study we demonstrated that DICER1 suppressed SUZ12 and EZH2 expression by directly affecting their upstream miRNAs in EC cells. In addition, knockdown of DICER1 promoted EMT and invasion of EC cells via histone hypermethylation and acetylation.

\section{Materials and Methods}

\section{Cell culture and transfection}

AN3 CA and Ishikawa cell lines were provided from Cell Bank of Chinese Academy of Sciences (Shanghai, China), and cultured in Dulbecco's modified Eagle's medium (DMEM)/F12 supplemented with $10 \%$ fetal bovine serum (FBS) 
(Gibco, CA, USA), $100 \mathrm{U} / \mathrm{mL}$ penicillin, and 100 $\mu \mathrm{g} / \mathrm{mL}$ streptomycin in a humidified atmosphere of $5 \% \mathrm{CO} 2$ at $37^{\circ} \mathrm{C}$. The plasmids encoding miR-200b and let-7i mimics or inhibitor and the control vectors, the plasmid encoding DICER1 siRNA (DICER1 siRNA) and the control vector (siRNA NC) were purchased from GenePharma (Shanghai, China). Cells were transiently transfected using Lipofectamine 2000 (Invitrogen, USA) according to the manufacturer's protocol.

\section{Patient specimens}

The endometrial carcinoma tissue samples were collected from patients who received hysterectomy at our hospital from April 2008 to March 2010. The histological aspects of the samples were judged following the criteria of International Federation of Gynecology and Obstetrics (FIGO) [14].

\section{Quantitative real-time PCR}

Total RNA was isolated from the cells or tissues using TRIzol (Invirogen). cDNA was synthesized using Prime Script RT Kit (TaKaRa, Dalian, China). PCR amplification was performed using TaqMan assay (Applied Biosystems, Foster City, USA) with the following primers: DICER1 Forward TTCCAAGTCGGTTGATACTGG, Reverse TGTTGATTGTGACTCGTGGAC; Vimentin Forward ATGCGTGAAATGGAAGAGAACT, Reverse CTCAGGTTCAGGGAGGAAAAGT; E-cadherin Forward GCTACTHHAACAGGGACACTTC, Reverse TTCTGCTGTGAAGGGAGATGTA; $\beta$-actin Forward CAG AGC AAG AGA GGC ATC C, Reverse CTG GGG TGT TGA AGG TCT C; SUZ12 Forward GGGGAAGTAGAACAGCAAAGAA, Reverse ACGGAGAGGTAAGCAGGTATCA; EZH2 Forward GATAAGGGCACAGCAGAAGAAC, Reverse ATCGCCTACAGAAAAGCGTATG. The relative mRNA levels were calculated using $2 \Delta \Delta$-Ct method.

\section{Western blot analysis}

Cells were collected and lysed in ice-cold RIPA buffer. Total protein in the lysate was quantitated and seperated by PAGE gels and transferred to the membranes. The membranes were washed and blocked with $5 \%$ bovine serum albumin for $1 \mathrm{~h}$, then incubated with primary antibody for DICER1 (1:1000 dilution, Abcam, UK), E-cadherin (1:1000 dilution, CST, USA) or $\beta$-actin (1:1000 dilution, CST, USA) at $4^{\circ} \mathrm{C}$ overnight. The membranes were washed and incubated with peroxidase-conjugated secondary antibody (1:5000) at room temperature for $1 \mathrm{~h}$. Finally, the membranes were developed using ECL kit and the signals were analyzed by using Total Lab software.

\section{Immunofluorescence}

Cells were cultured on glass cover slips, fixed and then permeabilized using $0.25 \%$ Triton X-100 at room temperature for $10 \mathrm{~min}$. The cells were incubated with $5 \%$ bovine serum albumin at room temperature for $1 \mathrm{~h}$, then incubated overnight at $4^{\circ} \mathrm{C}$ with primary antibody for DICER1 (1:50 dilution; Abcam, HK), E-cadherin (1:100 dilution; CST, USA), Vimentin (1:100 dilution; CST, USA) or $\beta$-actin (1:100 dilution; Epitomics, USA). Next the cells were washed and incubated with rhodamine-labelled secondary antibody (1:500 dilution, Epitomics, USA), then counterstained with DAPI. Control samples were incubated with PBS instead of primary antibody. Samples were mounted with antifade solution and analysed under laser scanning confocal microscope (Leica, Germany).

\section{Cell invasion assay}

$5 \times 10^{4}$ cells were loaded into the chamber of a Matrigel Invasion Chamber (BD Biosciences) with an $8 \mu \mathrm{m}$ pore size membrane. $48 \mathrm{~h}$ later the cells moved to the lower surface of the membrane were fixed by $100 \%$ methanol and stained with hematoxylin for cell counting.

\section{Global histone H3K27 and H3K4 methylation assay and total histone $\mathbf{H} \mathbf{3}$ acetylation assay}

$\mathrm{H} 3 \mathrm{~K} 4 / \mathrm{H} 3 \mathrm{~K} 9$ methylation and $\mathrm{H} 3 / \mathrm{H} 4$ acetylation were detected by using EpiQuik ${ }^{\mathrm{TM}}$ global histone H3/H4 acetylation and H3K4/H3K27 methylation kits (Epigentek, Farmingdale, NY, USA) according to the manufacturer's manuals.

\section{Statistical analysis}

All data were presented as mean \pm SD and analyzed by using SPSS 13.0 software. The differences were analyzed by one-way ANOVA for multiple-group comparison or $t$ test for two-group comparison. $P<0.05$ was considered significant.

\section{Results}

\section{The expression of DICER 1 in endometrial tissues and its association with clinicopathological parameters}

We assessed DICER1 expression levels in endometrial carcinoma tissue samples by IHC. We further detected the expression of DICER1 by qRT-PCR. As shown in Fig.1, from type II endometrial carcinomas to type I endometrial carcinomas, a increasing tendency of DICER1 expression was observed, and the difference among the three groups was significant $(\mathrm{P}<0.001)$, suggesting that the expression of DICER1 is associated with EC progression. 


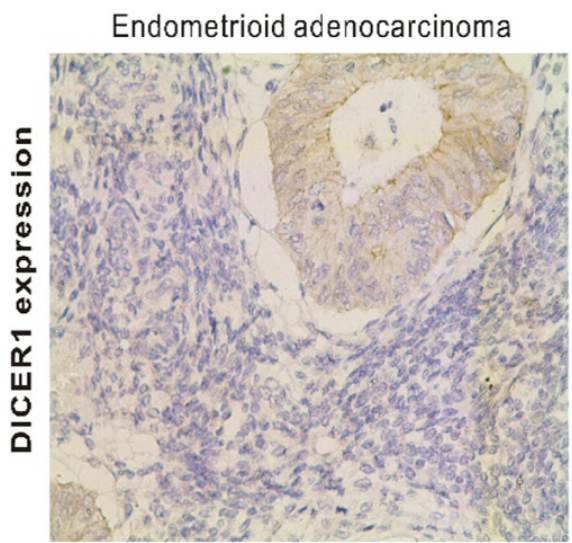

A

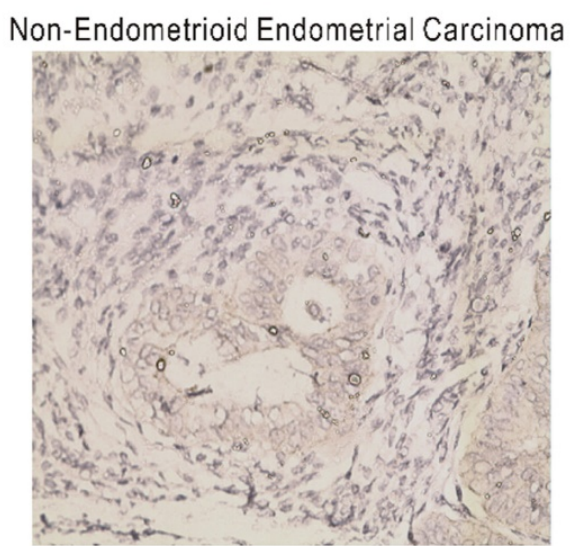

B

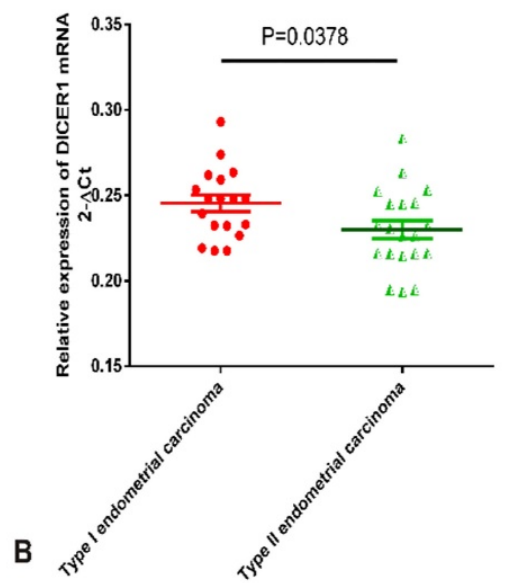

Fig. 1. DICER 1 expression in endometrial carcinomas. A. Immunohistochemistry analysis of DICER1 expression in endometrial carcinomas. Magnification: $x$ 400. B. qRT-PCR analysis of DICER 1 mRNA levels in clinical samples. DICERI expression was significantly higher in Type I endometrial carcinoma than in type II endometrial carcinoma.

\section{PRC2 components are targets of DICER I}

Our previous study demonstrated that DNA hypermethylation and histone deacetylation were implicated in the invasion of endometrial carcinoma cells [15]. Because PRC2 plays critical role in tumorigenesis and metastasis in many kinds of cancer, we next examined whether DICER1 could affect PRC2 in AN3 CA cells. In AN3 CA cells transfected with DICER1 siRNA, the expression of PRC2 components SUZ12 and EZH2 was upregulated significantly (Fig. 2A). In contrast, knockdown of DICER1 significantly downregulated miR-200b and let-7i. (Fig. 2B). Furthermore, miR-200b and let-7i mimics or inhibitor regulated SUZ12 and EZH2 expression levels, respectively (Fig. 2C). In addition, we showed that SUZ12 and EZH2 were targets of miR-200b and let-7i, respectively (Fig. 2D). Taken together, DICER1 could affect the expression of SUZ12 and EZH2 by modulating the level of their upstream miRNAs such as miR-200b and let-7i.

\section{DICERI suppresses EMT in vitro}

Next we evaluated the effects of DICER1 siRNA on EC cell invasion. AN3 CA cells transfected with DICER1 siRNA were significantly less invasive than control and untreated cells (Fig.3A). In addition, AN3 CA cells transfected with DICER1 siRNA showed increased E-cadherin expression accompanied by downregulation of Vimentin expression (Fig.3B). Furthermore, AN3 CA cells transfected with DICER1 siRNA exhibited typical EMT morphology, spindle-shaped appearance (Fig. 3C). Collectively, these data indicated that DICER1 could suppress EMT and the aggressiveness of EC cells.

\section{DICER1 regulates histone methylation and acetylation in EC cells}

Finally, we explored the role of DICER1 in histone methylation and acetylation in EC cells. Knockdown of DICER1 increased the level of total histone acetylation in Ishikawa cells. Moreover, we identified global histone H3K4 and H3K27 hypermethylation in DICER1 siRNA transfected EC cells, compared with control cells (Fig.4).

\section{Discussion}

DICER1 is an important enzyme of the miRNA machinery and may be a tumor suppressor in endometrial carcinoma. In our past research, we have found the correlation between DICER1 level and clinico-pathological features of endometrial carcinomas and found that DICER1 expression was significantly higher in FIGO I-II stages than in FIGO III-IV stage[13].

Here we found that dysfunction of DICER1 downregulated the expression of endometrial carcinoma related miRNAs including miR-200b and let-7i, which act upstream to regulate SUZ12 and EZH2 expression.

Epigenetic factors such as DNA methylation, H3K4me3, H3K27me3 and H3/4 acetylation are key regulators of gene expression in tumorigenesis. PRC2 is composed of SUZ12, EED and EZH1/2 and regulates the di-methylation and tri-methylation of histone $\mathrm{H} 3$ at lysine 27 to mediate transcription repression. We found that dysregulation of DICER1 may affect the expression of SUZ12 and EZH2, therefore regulating histone methylation and acetylation. Furthermore, H3K27 and H3K4 hypermethylation could lead to aberrant activation of cancer-associated genes such as E-cadherin. 

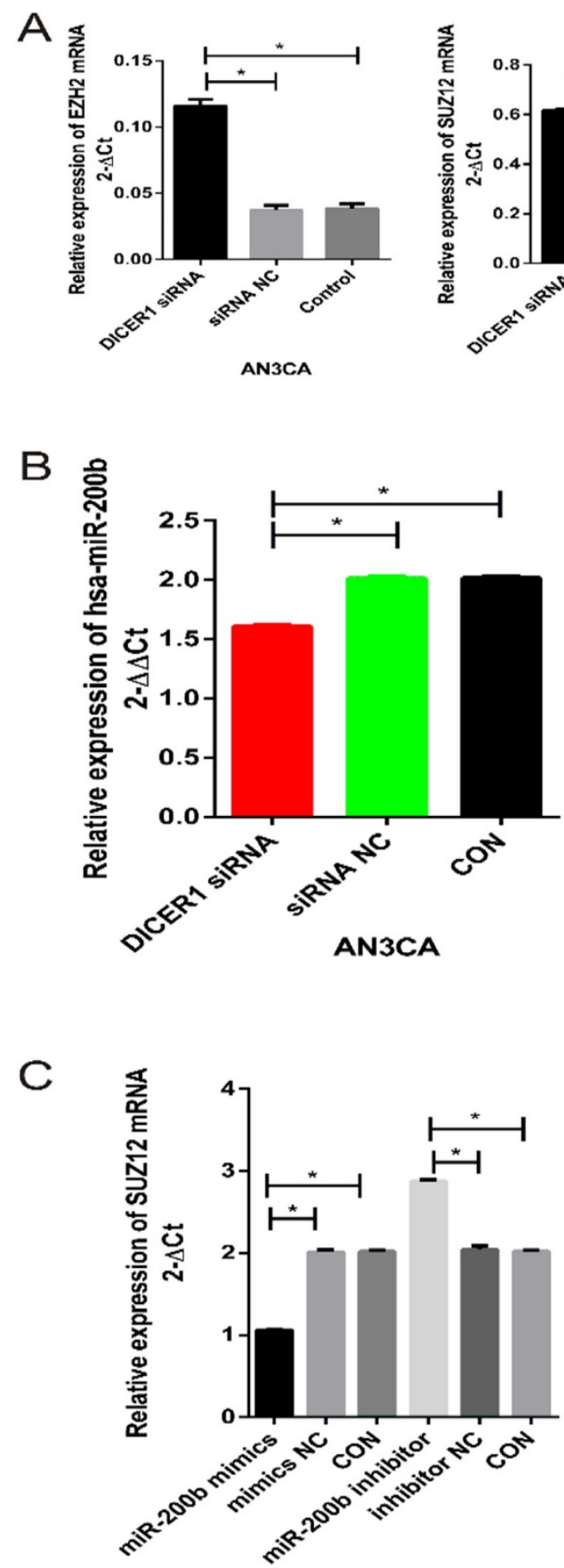

AN3CA
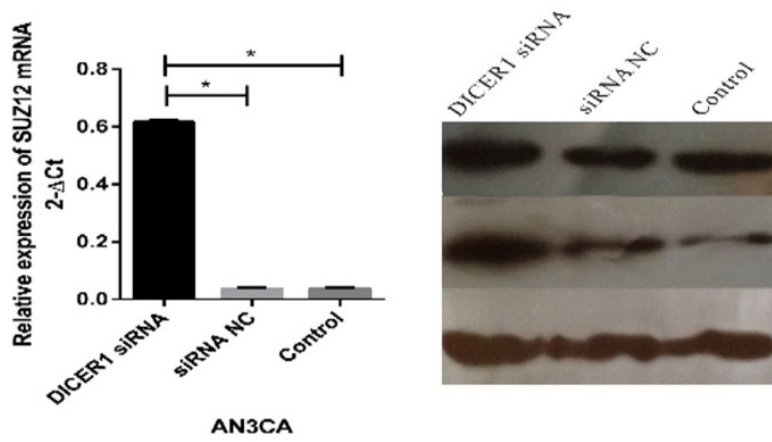

EZH2

SUZ12

B-actin

AN3CA
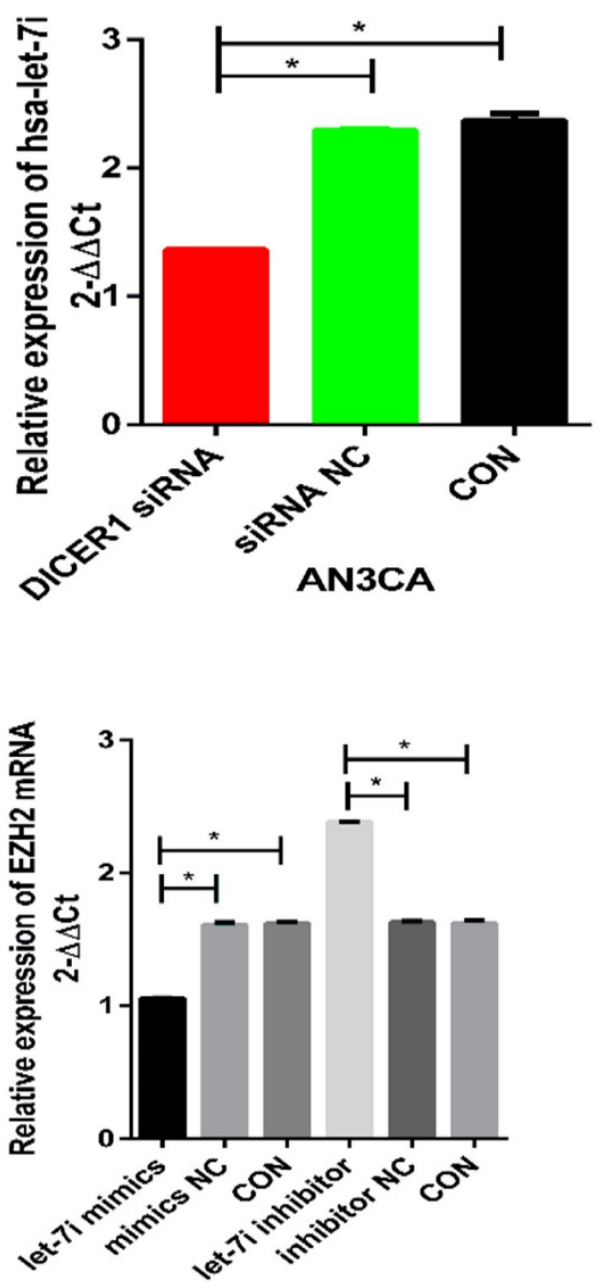

AN3CA

D C hsa-miR-200b/suZ12 Alignment

3. aguaguaaugguccgUCAUAAu $5^{\prime}$ ' hsa-miR-200b

232:5, 1111 ।

I I I I I I
AGUAUUY $3^{\circ}$ SUz12

mirSVR score: $\quad-0.1085$

PhastCons score: 0.6048

\section{C hsa-let-7i/EZH2 Alignment}

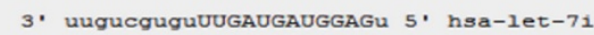

1:5, - I I I: I I I IIII

mirSVR score: $\quad-0.1592$

PhastCons score: 0.6879

Fig. 2. DICER1 suppresses PRC2 function in EC cells. A. qRT-PCR and Western blot analysis of SUZ12 and EZH2 expression in AN3 CA cells transfected with DICER 1 siRNA and NC. B. qRT-PCR analysis of miR-200b and let-7i expression in AN3 CA cells transfected with DICER1 siRNA and NC. C. qRT-PCR analysis of SUZ12 and EZH2 expression in AN3 CA cells transfected with miR-200b or let-7i mimics and inhibitor, along with their NCs. D. Complementary sequence between miR-200b and SUZ12 3'-UTR as well as between let-7i and EZH2 $3^{\prime}-$-UTR. ${ }^{*} \mathrm{P}<0.05$. 

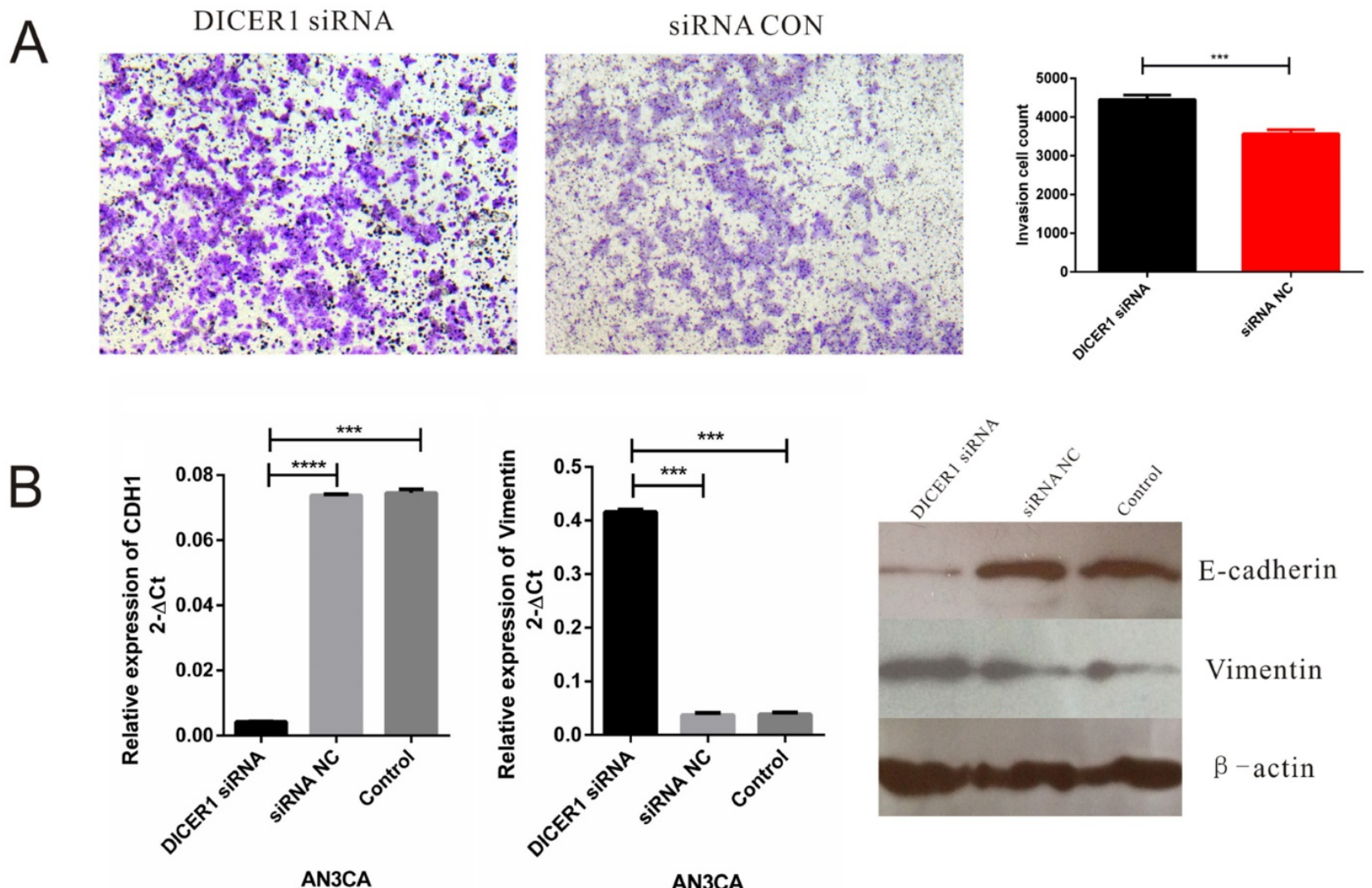

E-cadherin

Vimentin

$\beta-\operatorname{actin}$

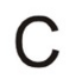

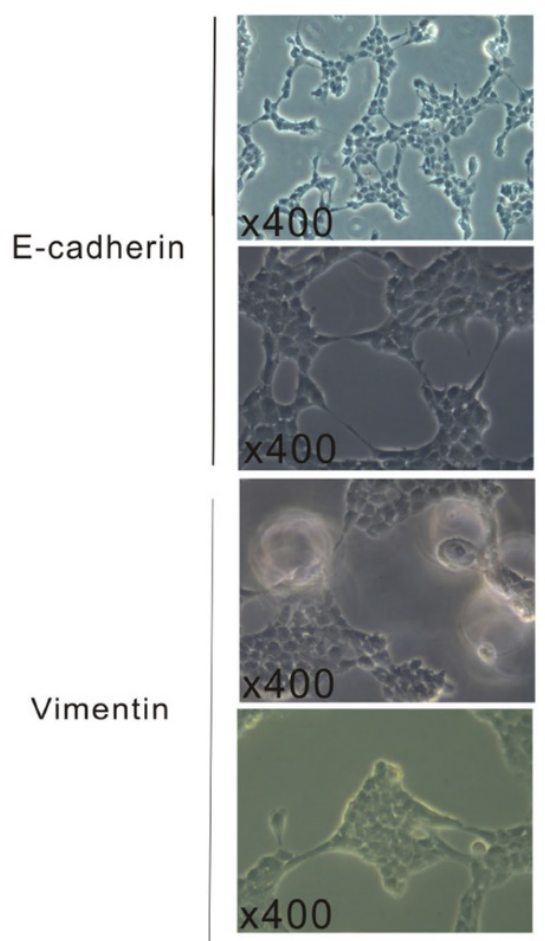

Bright field
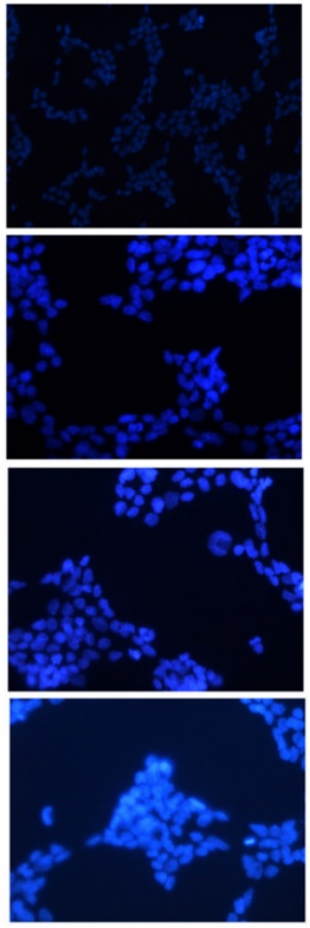

DAPI staining
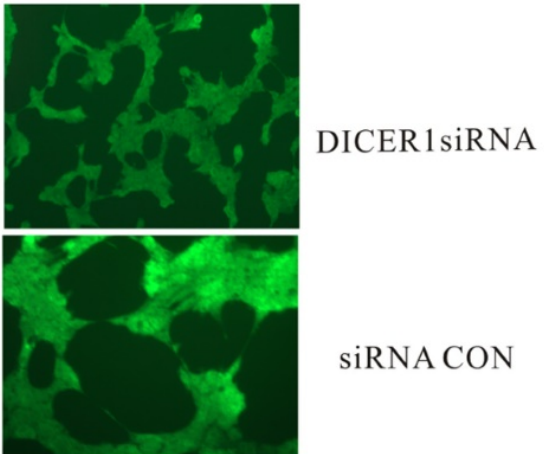

siRNA CON

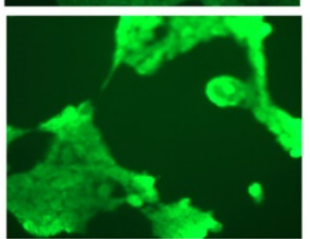

DICER1 1 iRNA

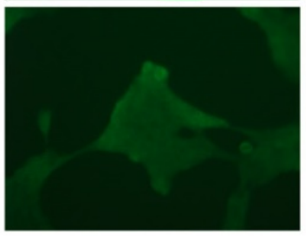

FITC staining

Fig. 3. DICER1 suppresses EMT in EC cells. A. Representative photos of transwell assay for AN3 CA cells treated with DICER1 siRNA. B.qRT-PCR and Western blot analysis of E-cadherin and Vimentin expression levels in AN3 CA transfected with DICERI siRNA and NC. C. Immunofluorescence microscopic analysis of the changes in the expression and localization of EMT markers. $* \mathrm{P}<0.05$. 
A

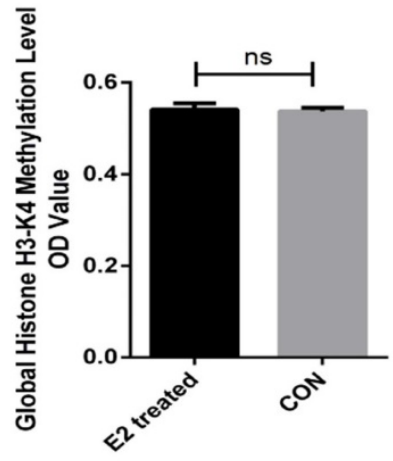

Ishikawa

B

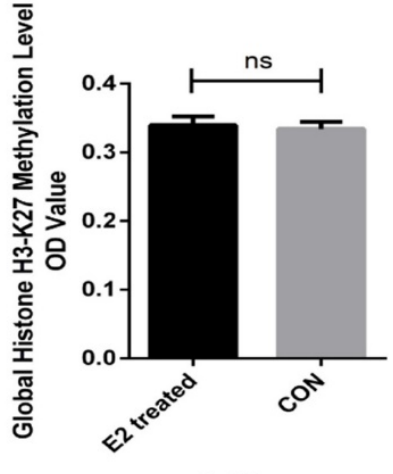

Ishikawa

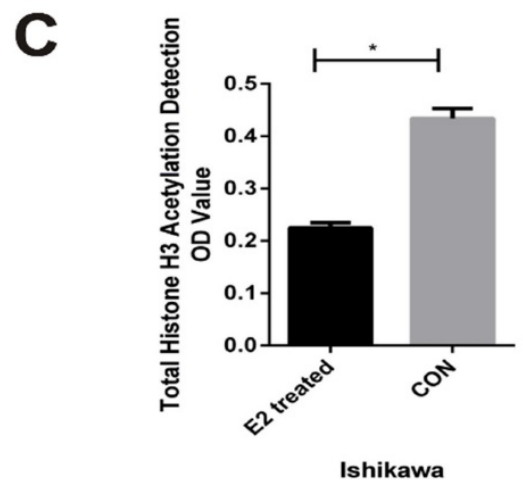

Fig. 4. DICERI affects histone methylation and acetylation in EC cells. A. Global H3K4 methylation levels in EC cells transfected with DICERI siRNA or treated with estrogen and their controls. B. Global H3K27 methylation levels in EC cells transfected with DICER1 siRNA or treated with estrogen and their controls. C. Global H3 acetylation levels in EC cells transfected with DICER1 siRNA or treated with estrogen and their controls. Methylation and acetylation levels were presented as the mean \pm SD. $* P<0.05$; ns: not significant.

We found differential patterns of global H3K4me3, H3K27me3 and total histone H3 acetylation between endometrial carcinoma cells transfected with DICER1 siRNA and untreated cells. Interestingly, DNA methylation and histone modification could crosstalk due to the interaction between SET domain of histone methyltransferases and DNA methyltransferases [16]. In this study, we found that DICER1-dependent miRNA regulation

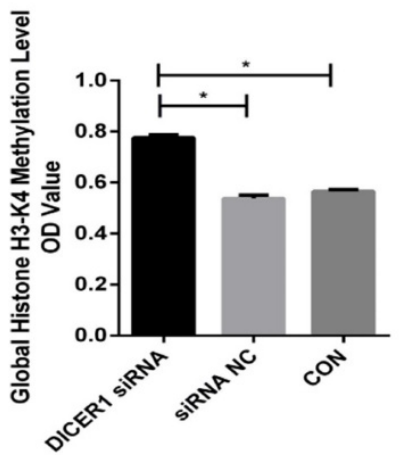

Ishikawa
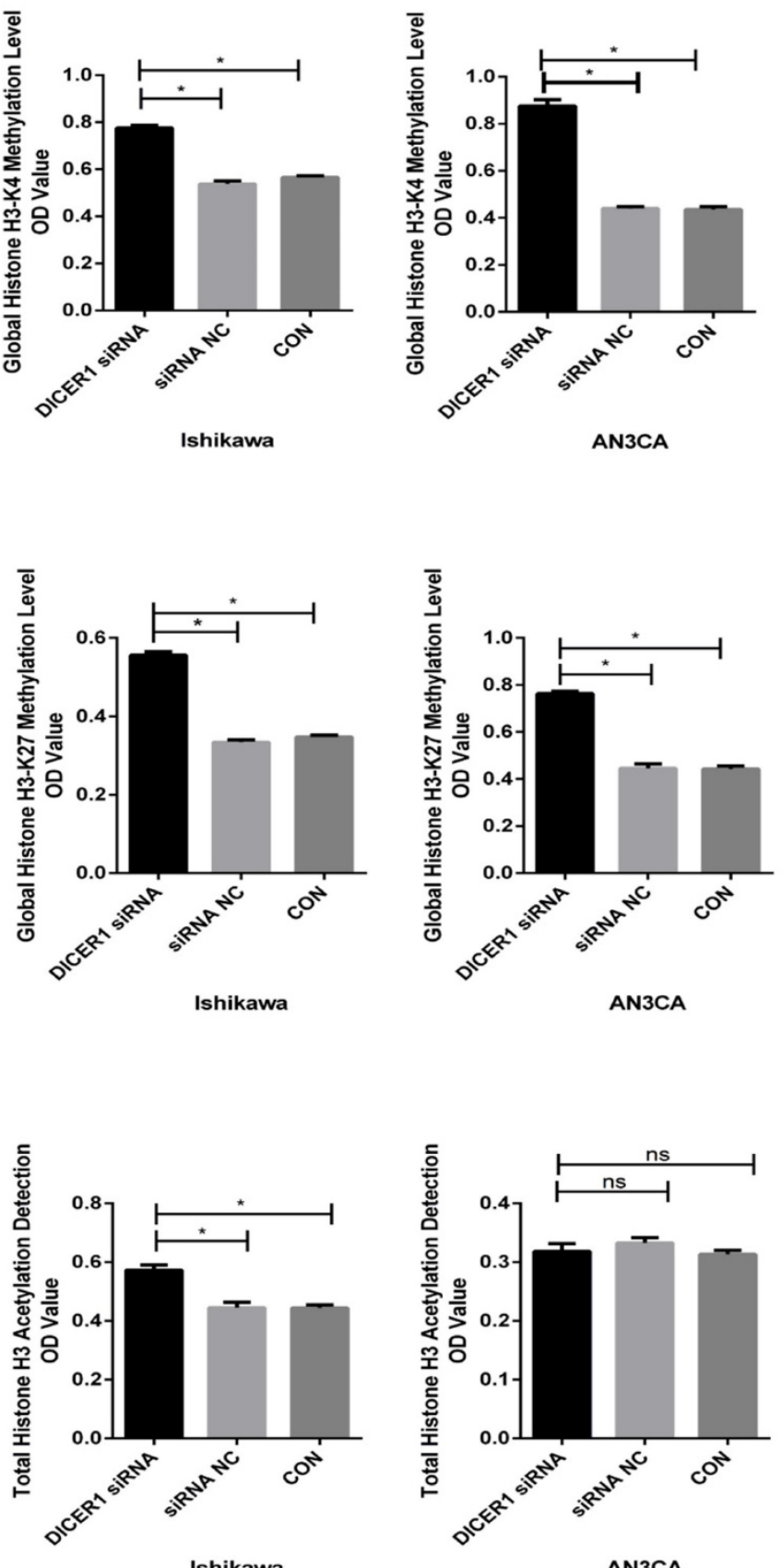

AN3CA

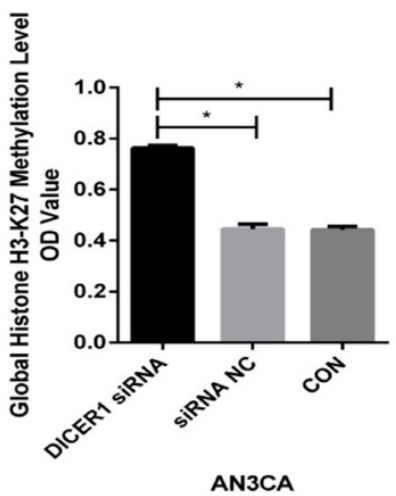

AN3CA leads to the changes in SUZ12 and EZH2 expression, which will then affect histone methylation levels. Thus we speculate that histone acetylation and DNA methylation form an epigenetic network to modulate the initiation and development of EC.

EMT is a multistage process that is required during embryonic development. However, increasing evidence suggests that EMT is often hijacked in various pathological conditions, such as tissue fibrosis 
and cancer metastasis $[17,18]$. We analyzed microRNA and EMT related signatures such as E-cadherin, vimentin, miR-200b and let-7i in EC cells. The results showed that DICER1 levels were correlated with the levels of E-cadherin, vimentin, $\mathrm{miR}-200 \mathrm{~b}$ and let-7i. These data suggest that the regulation of SUZ12 and EZH2 to modulate the level of global $\mathrm{H} 3 \mathrm{~K} 4 / 27$ and total $\mathrm{H} 3$ acetylation is implicated in EC invasion and metastasis.

Notably, epigenetic changes such as DNA methylation and histone modifications have emerged as new hallmarks of cancer. Since epigenetic modifications modulate gene expression without straightly modifying genetic sequences, reversing DNA methylation or histone modifications has potential for cancer therapy. To our knowledge, this is the first study to report epigenetic regulation of histone methylation and acetylation in EC cells. We provided evidence that DICER regulated certain EC-related miRNAs to induce aberration expression of PRC2 components SUZ12 and EZH2, leading to altered gene expression and malignant features of EC cells. Interestingly, low DICER1 mRNA levels were reported to be correlated with disease recurrence and worse DFS survival in EC patients [19]. Therefore, DICER1 is a promising epigenetic target for EC treatment.

\section{Acknowledgments}

This study was Sponsored by Natural Science Foundation of Shanghai (No.16ZR1427100), the National Natural Science Foundation of China (No. 81602280 and No.81602281).

\section{Competing Interests}

The authors have declared that no competing interest exists.

\section{References}

1. Siegel RL, Miller KD and Jemal A: Cancer statistics, 2015. CA: A Cancer Journal for Clinicians 2015; 65: 5-29.

2. Tsikouras $\mathrm{P}$, Bouchlariotou $\mathrm{S}$, Vrachnis $\mathrm{N}$, et al Endometrial cancer: molecular and therapeutic aspects. European Journal of Obstetrics and Gynecology and Reproductive Biology 2013; 169: 1-9.

3. Vialou V: Acétylation de la chromatine, régulation des gènes et dépression. Med Sci (Paris) 2010; 26: 465-467.

4. Schübeler D, Lorincz MC, Cimbora DM, et al. Genomic Targeting of Methylated DNA: Influence of Methylation on Transcription, Replication, Chromatin Structure, and Histone Acetylation. Molecular and Cellular Biology 2000; 20: 9103-9112.

5. Peterson CL and Laniel M-A: Histones and histone modifications. Current Biology 2004;14: R546-R551.

6. Costa FF: Non-coding RNAs, epigenetics and complexity. Gene 2008; 410: 9-17.

7. Verdone L, Agricola E, Caserta M and Di Mauro E: Histone acetylation in gene regulation. Briefings in Functional Genomics \& Proteomics 2006; 5: 209-221.

8. Wu Y, Strawn E, Basir Z, Halverson G and Guo S-W: Aberrant expression of deoxyribonucleic acid methyltransferases DNMT1, DNMT3A, and DNMT3B in women with endometriosis. Fertility and Sterility 2007;87: 24-32.

9. Margueron R and Reinberg D: The Polycomb Complex PRC2 and its Mark in Life. Nature 2011; 469: 343-349.

10. Bartel DP: MicroRNAs. Cell 2004;116: 281-297.
11. Kumar MS, Pester RE, Chen CY, et al. Dicer1 functions as a haploinsufficient tumor suppressor. Genes \& Development 2009; 23: 2700-2704.

12. Martello G, Rosato A, Ferrari F, et al. A MicroRNA Targeting Dicer for Metastasis Control. Cell 2010;141: 1195-1207.

13. Li B-L, Lu C, Lu W, et al. miR-130b is an EMT-related microRNA that targets DICER1 for aggression in endometrial cancer. Medical Oncology, 2013; 30: $1-13$.

14. Creasman W: Revised FIGO staging for carcinoma of the endometrium. International Journal of Gynecology and Obstetrics 2009;105: 109.

15. Li B-L, Lu W, Lu C, et al. CpG island hypermethylation-associated silencing of microRNAs promotes human endometrial cancer. Cancer Cell International, 2013; 13: 1-9.

16. Cedar $\mathrm{H}$ and Bergman $\mathrm{Y}$ : Linking DNA methylation and histone modification: patterns and paradigms. Nat Rev Genet, 2009; 10: 295-304

17. Thiery JP and Sleeman JP: Complex networks orchestrate epithelial-mesenchymal transitions. Nat Rev Mol Cell Biol, 2006; 7: 131-142.

18. Huang $\mathrm{Y}$, Zhao $\mathrm{M}$, $\mathrm{Xu} \mathrm{H}$, Wang $\mathrm{K}, \mathrm{Fu} \mathrm{Z}$, Jiang $\mathrm{Y}$, Yao Z. RASAL2 down-regulation in ovarian cancer promotes epithelial-mesenchymal transition and metastasis. Oncotarget, 2014; 5:6734-6745.

19. Zighelboim I, Reinhart AJ, Gao F, Schmidt AP, Mutch DG, Thaker $\mathrm{PH}$ Goodfellow PJ. DICER1 expression and outcomes in endometrioid endometrial adenocarcinoma. Cancer, 2011; 117:1446-1453. 